\title{
Design and simulation of a collision notification application with geocast routing for car-to-car communications
}

\author{
Ana María Orozco ${ }^{1} \cdot$ Sandra Céspedes $^{2,3} \cdot$ Roger Michoud $^{4} \cdot$ Gonzalo Llano $^{3}$
}

Received: 31 January 2015 / Accepted: 6 October 2015 / Published online: 27 October 2015

(C) The Author(s) 2015. This article is published with open access at SpringerLink.com

\begin{abstract}
Introduction Car-to-X Communications are envisioned to improve road safety, traffic efficiency, and information services through short-range and real-time systems. The enabling applications have varied requirements such as low latency, specific forwarding patterns, and reliable data exchange between vehicles and infrastructure. Hence, a cross-layer architecture for vehicular applications should be defined according to the goals of the implementation, so as to consider proper routing and dissemination mechanisms, communication protocols, and the application's design and operation.

Methods In this context, we propose the characterization of the upper layers of a safety-oriented Car-to-Car application. We propose a cross-layer application/network layer design for a Post Collision Notification (PCN) application, and by means of a coupled simulation model that combines the
\end{abstract}

This article is part of Topical Collection on the Role of Information and Communication Technologies (ICTs) in Facilitating Global Research, Development, and Technology Transfer Cooperation

Ana María Orozco

ana.orozco@tum.de

Sandra Céspedes

scespedes@ing.uchile.cl

Roger Michoud

rmichoud@cisco.com

Gonzalo Llano

gllano@icesi.edu.co

1 Technische Universität München (TUM), Munich, Germany

2 Department of Electrical Engineering, Universidad de Chile, Santiago, Chile

3 Department of ICT, Universidad Icesi, Cali, Colombia

4 École Polytechnique Fédérale de Lausanne, Lausanne, Switzerland communication network with vehicular traffic flow, we assess the performance of the application design.

Results Three main results can be derived from our work. First, the selected geocast protocol (DRG) shows to be an accurate protocol for safety applications and it may be a scalable routing mechanism for other applications. Second, that DRG is effective even for larger urban areas. And third, there is a reduction of acceleration and speed of the closest vehicles to the incident zone, which is a positive impact of the PCN application on the traffic flow.

Conclusions Consequently, the proposed cross-layer architecture and the implementation of geocast routing has shown a suitable support and good performance for safety applications, and could be extended to other application on the Car-to-X domain.

Keywords Car-to-car communications · Geocast routing · Collision warning application - Vehicular Ad Hoc Networks

\section{Introduction}

Vehicular Ad-Hoc Networks (VANETs) enable the wireless communications platform for Intelligent Transportation Systems (ITS) and its applications. VANET communication scheme allows vehicles and infrastructure to transmit real-time data in order to forewarn drivers about traffic situations, as well as mobility and environmental conditions. In this context, the safety sphere aims to reduce fatalities and mitigate damages caused by traffic accidents through Vehicle-to-Vehicle (V2V or C2C) applications and other ITS measures [1].

In the same way, collision warning applications have been proven to have an impact on drivers' behavior: benefits such as the improvement of the reaction times in incidents and the reduction of accidents rate have been shown in [2]. Similarly, Intersection Collision Warning Systems (ICWS) 
systems have shown to have an effect on intersection crashes by reducing on nearly a $50 \%$ the total number of collisions [3]. In this context, safety applications need to address accurate information with high reliability and low latency to the targeted dissemination zones; these requirements add complexity to the cross-layer architecture design of critical applications [4]. In particular, the vehicular networks, as a variant of the Mobile Ad-Hoc Networks, inherited the nature of the MANETs and consequently state novel design challenges. Regarding the characteristics associated with vehicular communications some important features can be listed: a highlyvariant topology, the self-organized and self-managed nodes behavior, the frequent network fragmentation, and the intricate radio communication aspects like unfavorable conditions for signal propagation (attenuation and reflection) and interference with other links. These particularities of the VANETs make it difficult to employ a traditional MANET network layer in the VANET, which is not completely suitable for this special category of networks. Therefore, the routing protocols involved in the data dissemination mechanisms must deal with these constraints [5]. This paper describes the cross-layer design of a Post Collision Notification (PCN) application and demonstrates its effectiveness based on the simulation of a geocast dissemination method. The main contributions of this paper can be summarized as follows:

- We propose a cross-layer architecture for the Post Collision Notification (PCN) application.

- We show the impact of the application on the mobility patterns of vehicles after the notification messages are disseminated by means of the Distributed Robust Geocast protocol.

- We conduct a set of simulations and obtain experimental results that demonstrate the effectiveness of the proposed design for the PCN application.

The reminder of this paper is organized as follows. Section 2 presents the related work. Section 3 introduces the characterization and design requirements of our Post Collision Notification application. Section 4 reviews the Distributed Robust Geocast routing protocol. Section 5 describes the simulation results and discussion. Finally, Section 6 concludes this paper.

\section{Related work}

A wide range of routing protocols and a diverse categorization for the vehicular communications has been considered in previous literature. Nevertheless, the decision-making process for deciding which criteria and routing schemas should be considered according to the VANET application is not a trivial task. A close examination of dissemination mechanisms leads to categorize routing protocols based on the destination target: unicast, multicast and broadcast [6]. Moreover, the variety of protocols offers a number of benefits for the implementation of vehicular applications. However, due to the nature of the collision notification applications, i.e., a strict low latency and a highly reliable communication scheme for a delimited area of interest, we focus this work on the revision of multicast routing, specifically on geo-based protocols or geocast; in this context, multicast can be considered an efficient data dissemination method for vehicular communications [7]. Correspondingly, according to Michoud et al. [8], the network layer of the VANET requires either adaptation of MANET protocols or new routing protocols design.

Furthermore, many location-based services demand the support for specific routing patterns of the information, namely spatial (n-dimensional geo-data) and temporal specifications [9]. In this context, position based routing is essential for vehicular applications, in which the geographical destination is a relevant decision criterion, as mentioned by Maihofer et al. [10]. In the safety sphere, Kihl et al. [11] proposed two redesigned geocast protocols: DRG and ROVER. Both protocols are based on the geographical location of the vehicle. Our PCN application implements a variation of the original Distributed Robust Geocast, which will be explained in section 4 .

Different from previous works, we propose the design and simulation of the Post Collision Notification application within a cross-layer architecture, by the implementation of Distributed Robust Geocast over the DSRC/WAVE standards. We also propose on this work a set of messages for upper layers called WaveAppMsg.

\section{Characterization of the post collision notification application}

The integration of the functionality and the effectiveness of the routing protocol in the VANET leads to model and couple the components of the logic (upper layer) and the network layer within a modular architecture. Our goal was to design a collision warning application and to adopt the most accurate dissemination method; to this end, we have developed a crosslayer architecture based on the requirements from the network and logical layers. This chapter describes the conceptual and modeling phases for the implementation of the PCN application.

\subsection{PCN application layer}

A Post Collision Notification application detects an incident based on the speed and deceleration of each vehicle or node. In this context, the term node refers to the vehicles on the adhoc network. After the identification of the incident, an 
accident-message is sent to the nodes in the region of interest. The PCN application is based on the communications standards defined for Wireless Access in Vehicular Environments (WAVE): IEEE 1609 [12] and IEEE 802.11p [13]. The design of the cross-layer architecture mostly depends on the intended target of the VANET application [14, 15]; for safety applications, the goal is to notify neighboring vehicles of hazard conditions (e.g. collision ahead); thus, the drivers can take actions previous, during, and before the emergency situations. For these types of applications the region of interest is commonly of medium-size (up to $1500 \mathrm{~m}$ ), and the messages require a high priority and a strict low latency communication (typically less than $100 \mathrm{~ms}$ ).

The functionality of the logical layer of PCN is described below:

- Parameters initialization: The application layer of the PCN is responsible of the initial phase, including parameters definition, such as: operational range of the application (in miles or kilometers), beacon intervals (in seconds) and the connection establishment with the Global Positioning System (GPS) or urban mobility simulator.

- Data encapsulation: We propose a set of messages, so called the WaveAppMsg, following the modular design. A WaveAppMsg packet contains an identification number (ID), the header field, the data and an optional field. The data correspond to the geographical coordinates of the emergency event; for PCN, an additional field is employed to indicate the accident status, showing that either the accident is ongoing or it has been successfully resolved.

- Accidents detection: To avoid collisions with new arrival vehicles in the scene of a previous accident, PCN detects and notifies when there is a stopped vehicle on the road due to an accident. According to the speed and deceleration, PCN calculates an abruptly halt and triggers the notification event called accident signal.

- Triggering of the sending mechanism: PCN starts the data dissemination process once the event trigger-accident signal-is launched right after the accident's detection. This layer also defines the periodicity and the amount of packets to send through the VANET.

- Vehicle position retrieval: PCN requires the up-to-date geographical location of the vehicle, so as to deliver reliable information about an occurred accident within actual coordinates. The application layer employs geo-data from a GPS or a road network simulator.

- Data storage: Once data are delivered to the destination, each recipient node stores the information related to the accident. The reason is to update nearby drivers about road events and to maintain traffic data for statistical and mobility purposes.

\subsection{Network requirements for PCN}

The design of vehicular applications also involves the identification of the properties associated to the network model. These aspects must be redefined with regards of the requirements for each application. This section describes the characterization process for the routing layer for PCN application. Table 1 summarizes the requirements for the application design. Moreover, the key criteria are listed bellow:

- Participants: it specifies if it is a Vehicle-to-Vehicle (V2V) or a Vehicle-to-Infrastructure (V2I) communication.

- Latency: it defines the total delay experienced from the time the packet is sent by the source to the moment it is delivered to the recipient: low $(<100 \mathrm{~ms})$ and medium ( $>$ $100 \mathrm{~ms}$ ). This parameter provides message priority information.

- Region of interest: it describes the geographical range of the application: long $(>1500 \mathrm{~m})$, medium $(\sim 1000 \mathrm{~m})$ and short $(<500 \mathrm{~m})$.

- Geo-location: it describes if the vehicle's position in the geographical coordinate system is necessary to perform any data process.

- Recipient pattern: it describes the message receiver's pattern. In other words, it says to whom the messages emitted have to be transmitted. The pattern can be one-to-many, one-to-a-zone, one-to-one, and many-to-one.

- Trigger: it states how the application is triggered. There are three different modes; Beacon mode (periodic), eventtriggered mode (event driven), and user-initiated-ondemand mode (user-initiated).

- Routing protocols: this network attribute is highly related to the Recipient pattern criterion. There are four different categories: broadcast (one-to-many) and geocast (one-to-a-zone) for safety applications, and unicast (one-to-one) and aggregation (many-toone), which are more suitable for commercial

Table 1 PCN Application requirements

\begin{tabular}{ll}
\hline Parameter & Value \\
\hline Participants & Car-to-Car (V2V) \\
Max. End-to-End delay & Low $(<100 \mathrm{~ms})$ \\
Infrastructure & Not required \\
Region of interest & Medium $(<1500 \mathrm{~m})$ \\
Geo-location & GPS/Simulator \\
Recipient pattern trigger & One-to-a-zone \\
Time-to-live & Event-triggered \\
Routing protocol & Multi-hop \\
Packet format & Geocast \\
Channel frequency & WAVE Short Message (WSM) \\
& Control Channel (CCH) \\
\hline
\end{tabular}


applications. As we want to cover the direct neighbor of the crashed vehicle with PCN messages, we will favor the geocast routing scheme.

- Message packet format: it specifies the network packets format used to encapsulate application messages. There are two standards from which the application's designer can choose, the Internet Protocolv6 (IPv6) or the WAVE Short Message Protocol (WSMP) [12].

\section{Geocast routing scheme for the PCN application}

A common problem to overcome in most VANET applications is the low reliability of the network, caused in many cases, by the highly variable topology of the mobile ad hoc network.

Improvements of the reliability of the vehicular networks can be handled by the proper routing mechanism on the VANET. In other words, the reliability relies on an effective routing method and the forwarding techniques. For this sake, multi-hop and position-based multicast are implemented to cover geographic regions while assuring packet delivery to all nodes in the area of interest. Distributed Robust Geocast (DRG), a geo-based routing protocol, is a completely distributed and stateless protocol for a reliable location-based dissemination with no control overhead.

The purpose of using "selective broadcast" is to limit the flooding area to a specific zone, based on geo-data namely geographical coordinates; this routing decision minimizes the network load in contrast to the broadcast storm caused by a generic broadcast scheme. As shown in Table 1, the PCN application requires a multi-hop delivery mechanism in order to cover a delimitated area. For these reasons, DRG was considered the suitable routing protocol for the network layer of our PCN application.

\subsection{DRG protocol aspects}

Geocast is a variant of multicast routing, where the multicast group comprises the set of all nodes belonging to the targeted geographical region. The geocast routing scheme forwards the packets from a unique source to a defined geographical area [16]. DRG works under the following assumption: if a node is located within the targeted geographic region, it processes the packet; otherwise, it just drops it. In order to implement this protocol, two principles are employed [17]: the Zone Of Relevance (ZOR) and the Zone Of Forwarding (ZOF). The $\mathrm{ZOR}$ is the proper geographical area to cover, whereas the ZOF is used in order to ensure that the messages will reach every node in the ZOR. All the nodes present in the ZOF forward the messages to vehicles present in ZOR. Usually, the ZOF surrounds the ZOR. This mechanism is implemented in order to overcome the highly dynamic topology problem of the moving nodes on the road network. This approach aims also to control the typical flooding problem in a traditional broadcast scheme. Another benefit of DRG is that the source node periodically re-transmits the messages to the ZOF; thus, the network fragmentation problem is solved. Figure 1 shows the ZOR and the ZOR definition in a road network scenario. For PCN, we have introduced a set of message that allows DGR to acknowledge the type of events, the time to live, and also the geographical area to forward the notification messages in the zone of interest.

\subsection{Forwarding mechanism of DRG}

The application layer triggers the forwarding mechanism of DRG once an accident is detected; afterward, the PCN application layer sends a PcnAccidentMsg to the lower layer containing the geographical coordinates of the emergency (position of the node which detected the incident) and the information in regards the incident. Then, the source node creates a WAVE Short Message Packet (WSMP) at the level of the network layer, which encapsulates the geographical coordinates, the incident status and the radius of the zone of relevance. When the nodes within the zone of forwarding receive a WSPM, the network layer unpacks the data and calculates the Euclidean distance for a two-dimensional plane (2-d geographical coordinate system). After the emergency event has been resolved and the area is cleared, PCN notifies the vehicles within the zone of relevance. The PCN application's upper layer generates a PcnAccidentResolved message, which contains the coordinates of the event and the new accident status. The dissemination method for PcnAccidentResolved

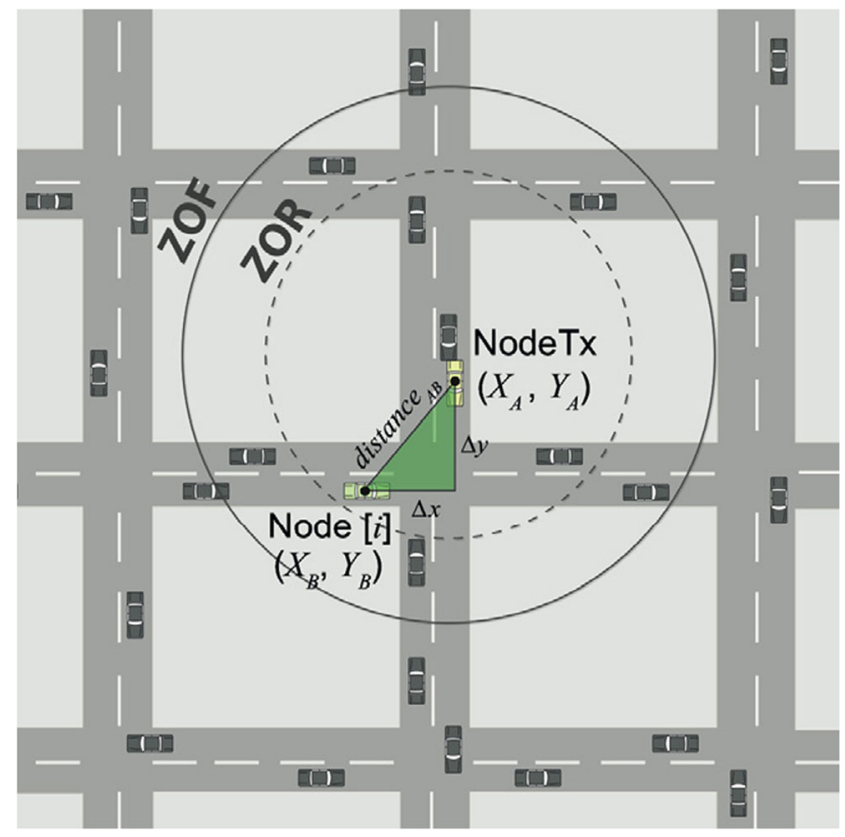

Fig. 1 DRG protocol scheme denoting ZOF and ZOR 
messages employed is the same scheme of DRG. The communication process is depicted in Fig. 2.

\section{Simulation tools and ICT for vehicular communications research}

\subsection{Simulation setup of PCN application on the V2V domain}

The simulation of vehicular communications is a valuable tool to assess the performance, requirements, and feasibility of the VANET applications. Since the implementation of many cooperative systems require high costs, a complex infrastructure and a high level of coordination and logistics, it is very common that these projects are first evaluated using a simulation approach. Recent literature of VANETs refers to hybrid simulation as the most accurate technique to analyze Inter-Vehicle Communications (IVC) [18]; for this sake, coupling a network and microscopic traffic simulator provides a representative model of the mobility and communication patterns in urban scenarios [19].

We have performed a cross-layer simulation, which includes the physical; media access control (MAC), and the routing and application layers to evaluate the performance of our design. Considering this approach, we want to validate three concepts: First, that DRG is an accurate protocol for safety applications with low latency demand, and that it is a scalable routing mechanism for other applications with similar dissemination requirements. Second, that DRG is effective even for larger urban areas. And third, what is the impact of the PCN application on road traffic, once an accident has been detected and the neighbor drivers are successfully notified. An accurate integration of the application functionality and the effectiveness of the routing protocol leads to the success of the application, hence, the applications can positively influence the traffic flow and the drivers' behavior and their interactions.

\subsection{Simulation scenario}

The simulation is built upon Veins [18], an open source vehicular network simulation framework. Veins makes use of the interface called Traffic Control Interface (TraCI) of traffic simulation suit SUMO Simulation of Urban MObility [20] and $\mathrm{OMNeT}++$ [21]. Our city-scenarios and geo-data come from the OpenStreetMap project, an open data repository for geographical information. The microscopic model employed is the car-following model proposed by Krauss [22], this model allows the simulation of platooning of vehicles and reproduces the drivers' behavior. This model provides the driver's perfection parameter $\sigma$ to adjust the accuracy of the drivers' behavior; in our scenario $\sigma$ was set to 0.5 to add dawdle to the drivers' responsiveness; furthermore, the accidents have been manually set up.
Fig. 2 Cross-layer design of PCN

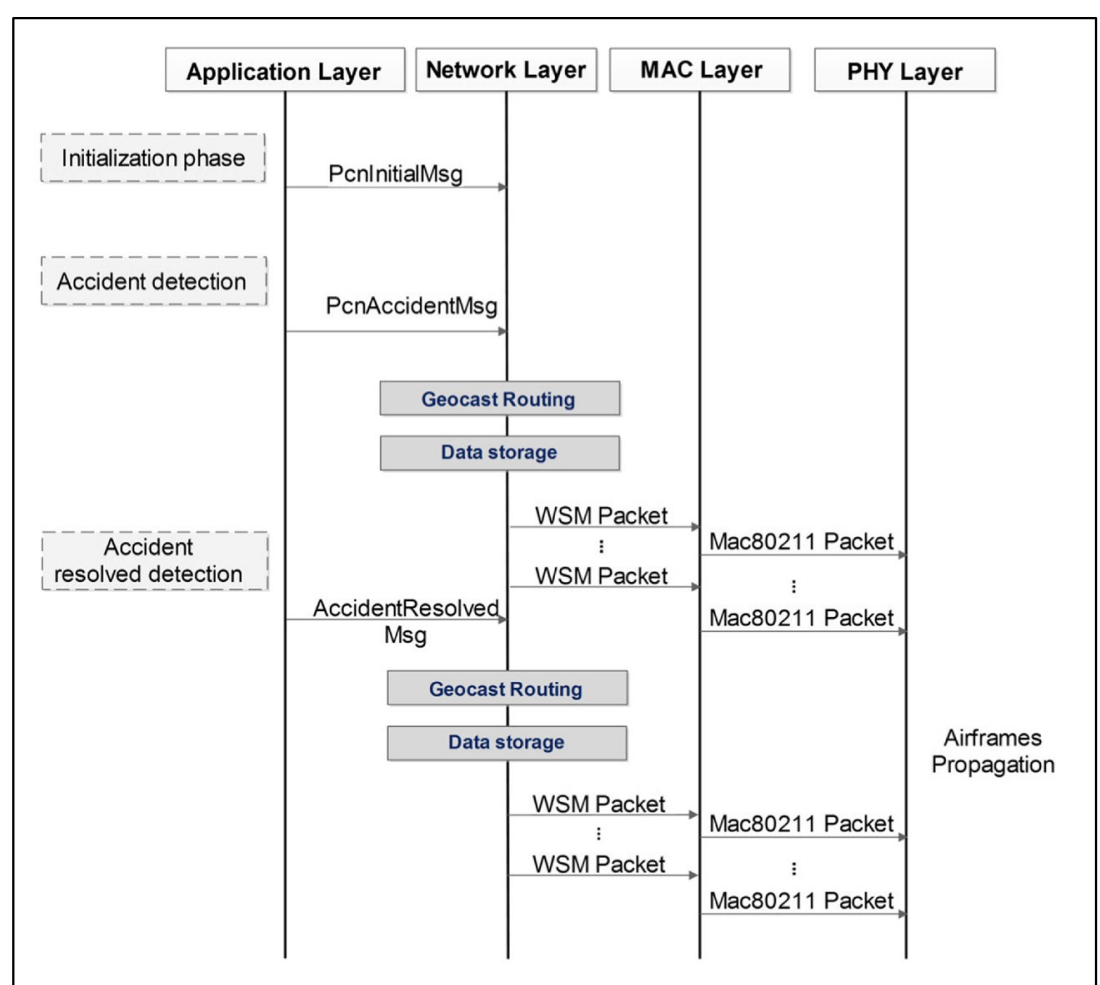


Table 2 Mobility Model Parameters

\begin{tabular}{lll}
\hline Parameter & Car & Truck \\
\hline Acceleration $\left(\mathrm{m} / \mathrm{s}^{2}\right)$ & 2,6 & 2,6 \\
Deceleration $\left(\mathrm{m} / \mathrm{s}^{2}\right)$ & 4,5 & 3,5 \\
Max. Speed $(\mathrm{m} / \mathrm{s})$ & 14 & 11 \\
Min. Gap $(\mathrm{m})$ & 3 & 3 \\
Length $(\mathrm{m})$ & 3 & 5 \\
Driver's Dawdle $\sigma$ & 0,5 & 0,5 \\
\hline
\end{tabular}

We have deployed vehicles of two types: cars and trucks. Table 2 depicts the road traffic parameters and describes characteristics of the car and truck traffic flows. For the lower layers of the wireless communication model we have employed the 802.11p MiXiM package, which implements the Dedicated Short Range Communications (DSRC) standard. We have considered all vehicles equipped with 802.11p DSRC radio on board and we assumed that all vehicles participate in the ad-hoc network. The Car-to-X (C2X) communication parameters are summarized in Table 3.

Two metrics have been selected in order to assess the performance of the geocast routing mechanism: End-To-End Delay and Packet Reception Rate (PRR).

- End-to-End Delay: it is the time elapsed since a packet is sent by the application layer at the source node until the recipient node's application layer receives the packet [23]. The latency is a common metric used to show the effect of larger areas to cover and also the impact of nodes on the performance of the protocol.

- Packet Reception Rate (PRR): it is defined as the percentage of nodes that successfully receive a packet from the tagged node given that all receivers are within transmission range of the sender at the moment the packet is sent

Table 3 Simulation Framework Parameters

\begin{tabular}{ll}
\hline Parameter & Value \\
\hline Application & \\
Beacon interval & $0,1 \mathrm{~s}$ \\
Routing data length & $320 \mathrm{bit}$ \\
Max. power & $20 \mathrm{~mW}$ \\
Connection Mng daturation & $-89 \mathrm{dBm}$ \\
Mac carrier frequency & $5,890 \mathrm{e} 9 \mathrm{~Hz}$ \\
Mac. 1609.4. Tx power & $20 \mathrm{~mW}$ \\
Mac1609.4. bitrate & $18 \mathrm{Mbps}$ \\
Phy 80211p sensitivity & $-89 \mathrm{dBm}$ \\
Phy 80211p. Max Tx power & $10 \mathrm{~mW}$ \\
Antennas' high & $1895 \mathrm{~m}$ \\
Simulation area & $4500 \mathrm{~m} \times 6000 \mathrm{~m}$ \\
\hline
\end{tabular}

out [24]. It is defined by Khan et al. [25] as a percentage given by:

$P R R_{(Z O R)}=\frac{\sum \text { NodesReceivingDataPackets }}{\sum \text { NodesWithinTheZOR }}$

\subsection{Simulation results}

As mentioned before, the critical latency for safety applications is up to $100 \mathrm{~ms}$. Figure 3 shows the latency obtained for diverse coverage areas that vary from $500 \mathrm{~m}$ to $4000 \mathrm{~m}$; the impact on the end-to-end delay can be observed, as the zone of relevance is larger. Larger distance to the intended receivers require more hops, therefore it takes longer to propagate the messages over the network. It is important to mention that even though the latency increments, the nodes up to $4000 \mathrm{~m}$ are receiving the geocast packets within the expected time for the PCN application or other safety applications. This reflects the efficiency of the protocol for safety applications. We observe that for $1500 \mathrm{~m}$ - the zone of relevance for most safety applications - the efficiency is an $85 \%$ lower $(14.85 \mathrm{~ms})$ than the maximum delay accepted at the ZOR.

The effect of the traffic density is depicted in Fig. 4. It can be observed that the end-to-end delay gradually increases as the number of nodes increments, which is an expected behavior. When the traffic is denser, DRG meets the expected delay requirement - less than $100 \mathrm{~ms}$ - in the zone of relevance for PCN application (1500 m). Moreover, the behavior of the endto-end delay is similar to the latency variation as a function of the zone of relevance shown in Fig. 3, where large distances do not significantly affect the end-to-end delay; these characteristics show DRG to be a stable protocol, because it

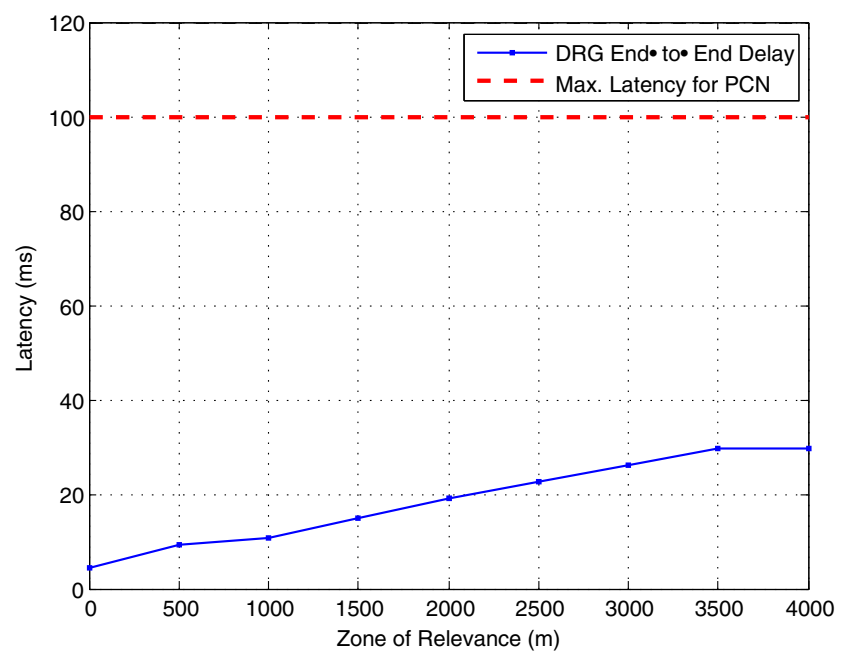

Fig. 3 End-To-End Delay for PCN with variation of the Zone of Relevance 


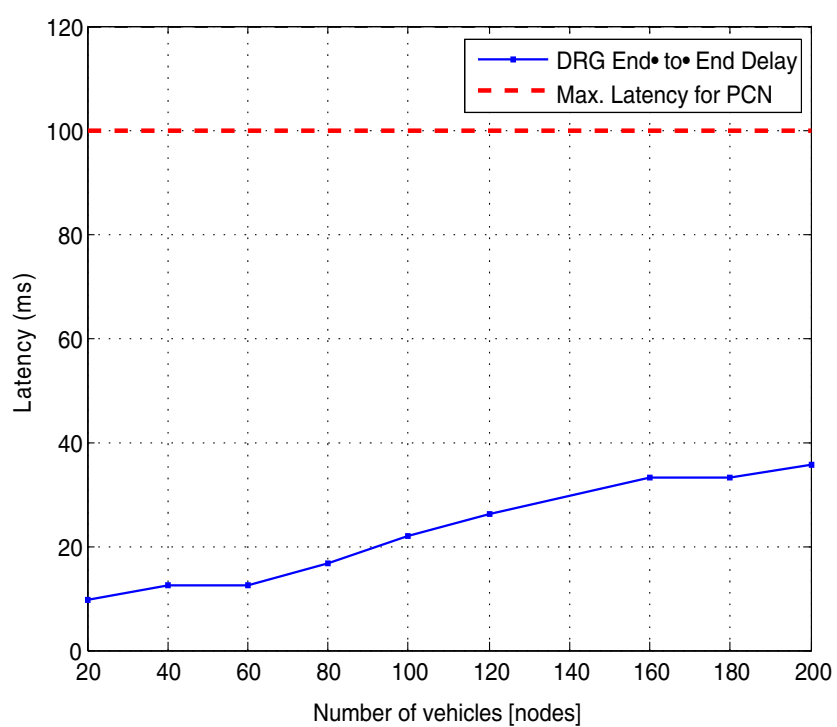

Fig. 4 End-To-End Delay for PCN with variation of the number of nodes within the Zone of Relevance

maintains a high performance for an incremental variation of the nodes density and the size of the zone of relevance.

The PRR has been compared versus the single hop broadcast scheme, where the maximum range for simple broadcast is up to $300 \mathrm{~m}$. It can be observed in Fig. 5 that, when the zone of relevance is set as $500 \mathrm{~m}$, the PRR achieved $100 \%$, and for $1500 \mathrm{~m}$, the PRR decreases slightly to $98.8 \%$, showing a suitable performance for collision warning applications like our proposed PCN application. For larger distances, between $1500 \mathrm{~m}$ to $3500 \mathrm{~m}$, the PRR gradually decreases as expected. Further, when the ZOR is set to $4000 \mathrm{~m}$, the PRR is still near to $80 \%$, which is considerable for traffic applications demanding a bigger area of interest to cover. We have also tested the impact of the density $k(v e h / \mathrm{km})$ on the PRR for a zone of relevance set to $1500 \mathrm{~m}$. The traffic density is a determinant macroscopic variable, which gives an idea of the degree of

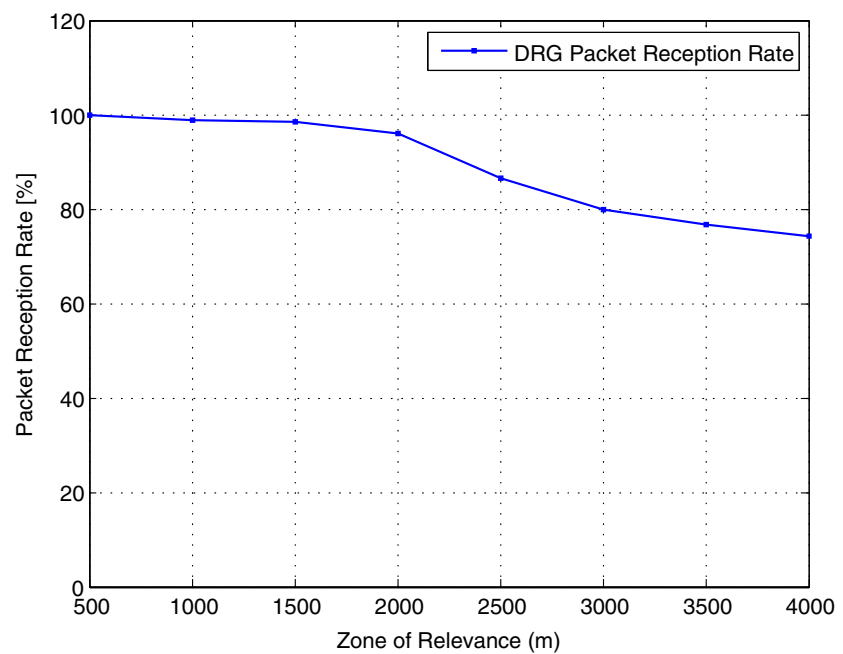

Fig. 5 Impact of ZOR variation on the Packet Reception Rate PRR for PCN

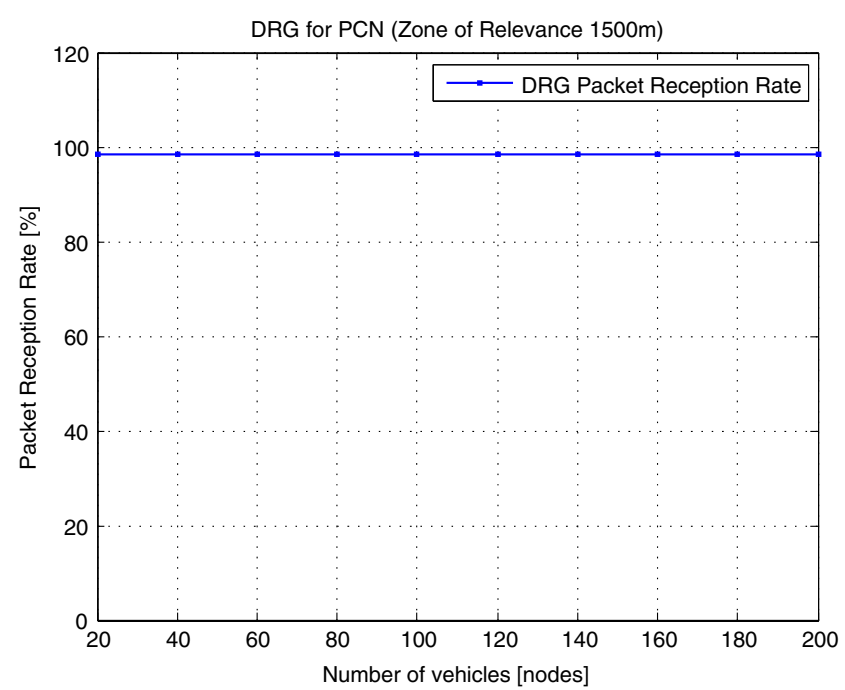

Fig. 6 Impact of the increment of the node density on the PRR

congestion of a road segment when it is analyzed in combination of the flow volume and average speed of the traffic stream. In this case, Fig. 6 shows that the increment of the number of nodes does not affect the PRR, which maintains a constant value of $98.8 \%$.

The performance of PCN application has been evaluated through the PRR also as a function of velocity, in order to validate the effectiveness of the application for different vehicular velocity. Fig. 7 shows the impact of the velocity of the vehicles in the zone of relevance. For urban areas where the typical speed is $60 \mathrm{Km} / \mathrm{h}$, DRG protocol shows high rate of the recipient nodes up to $80 \mathrm{~km} / \mathrm{h}$. It can be observed that vehicles do not affect the PRR until $80 \mathrm{~km} / \mathrm{h}$; which means that drivers on urban roads and highways effectively received PCN notification messages.

We have assessed the velocity and acceleration of the vehicles nearby the accident's location to show how the speed

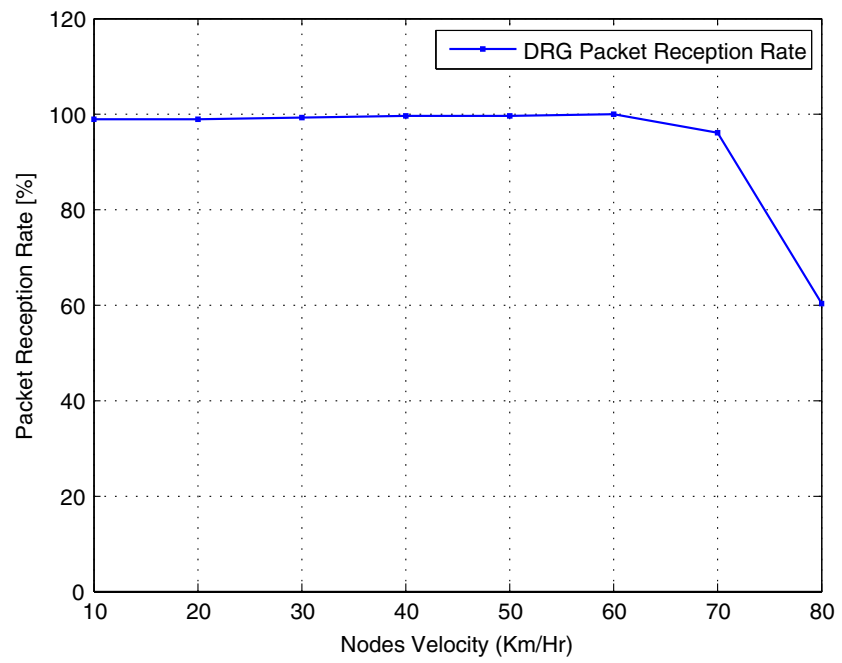

Fig. 7 Impact of velocity of vehicles on the Packet Reception Rate PRR for PCN application 
Fig. 8 Effect of the VANET application on vehicles' speed

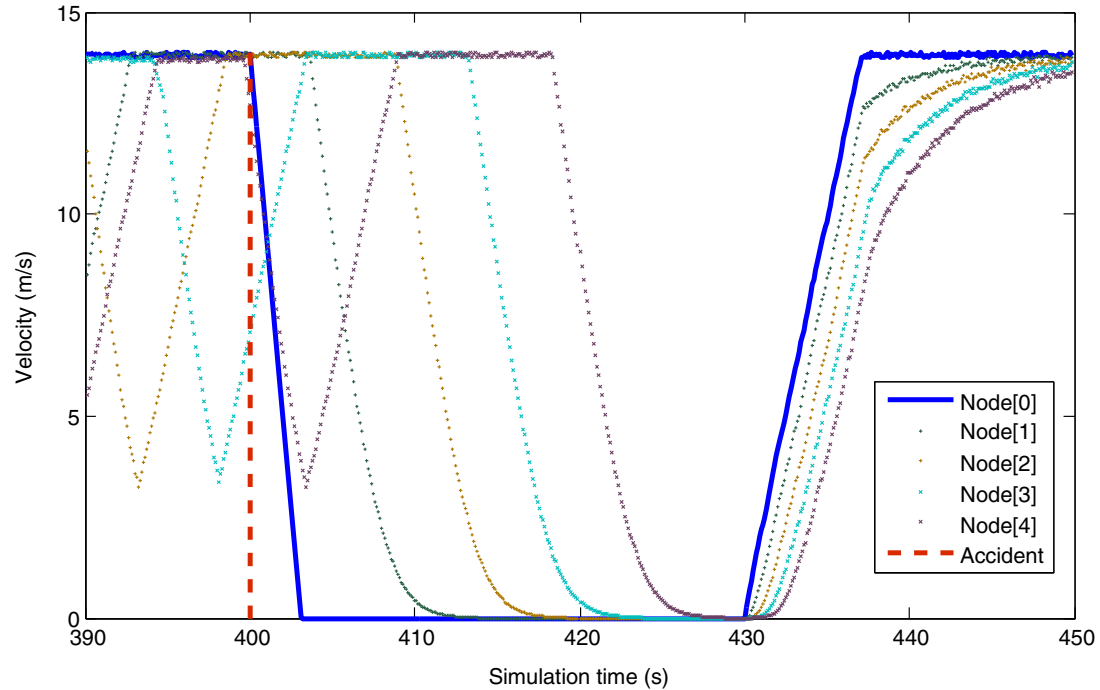

varies after the reception of the WaveAppMsg packets of the PCN application. Therefore, we have measured the variation of velocity as a function of the simulation time. Fig. 8 shows five nodes in the period of time starting in $390 \mathrm{~s}$. At $400 \mathrm{~s}$, the first node on the road (Node 0) abruptly stops, which is detected by the application layer as an accident, and it triggers the DRG forwarding mechanism. Nodes 1 to 4 receive the PcnAccidentMsg packets and decrease their speed until they completely stop. After $30 \mathrm{~s}$, the accident is solved and Node 0 sends PcnAccidentResolvedMsg packets at the $430 \mathrm{~s}$, then Nodes 1 to 4 accelerate and traffic returned to normal. Such a behavior demonstrates the effectiveness of the PCN design, regarding the harmonization of the velocity and acceleration of the vehicles within the zone of interest. The speed of the stream is gradually reduced, in contrast to the first vehicle, the acceleration of the vehicles in front the incident also decreases smoothly. It can be assumed that after the drivers received the notification messages (few seconds after the incident detection) the break system was activated; Fig. 8 demonstrates the aforementioned behavior.

\section{Conclusions}

In this work, we have proposed a cross-layer design of a collision warning application coupled with a distributed geocast routing mechanism. We have characterized the application and network layer in order to achieve a high delivery ratio and low latency. Since the network layer is responsible of data dissemination, we have chosen and adapted the Distributed Robust Geocast scheme as the routing protocol. The implementation of geocast for our PCN application has shown a suitable support and good performance for safety application. The packet reception rate of DRG has shown to not be sensitive to the number of nodes and the zone of relevance.
The results also show that DRG can be employed for other applications, due to its scalability and stability, either for larger distances or for higher densities of vehicles within the zone of interest. Other applications with similar routing requirements such as the Stopped/Slow Vehicle Advisor (SVA), Emergency Electronic Brake Light (EEBL), Road Hazard Condition Notification (RHCN), Road Feature Notification (RFN) and Congested Road Notification (CRN) can be de- signed considering similar requirements in the network layer, and may implement DRG for data dissemination.

For our future work, we will test the cross-layer design with other safety-oriented applications that are also dependent on the geographic location. Furthermore, we would like to design other applications oriented to influence the traffic flow and to improve the vehicular traffic efficiency.

Acknowledgments This work is partially funded by CONICYT Chile through Project FONDECYT No. 11140045. Authors would like also to thank Colciencias (Administrative Department of Science, Technology and Innovation, Colombia) for holding this project under the program "Young Researchers and Innovators".

Open Access This article is distributed under the terms of the Creative Commons Attribution 4.0 International License (http:// creativecommons.org/licenses/by/4.0/), which permits unrestricted use, distribution, and reproduction in any medium, provided you give appropriate credit to the original author(s) and the source, provide a link to the Creative Commons license, and indicate if changes were made.

\section{References}

1. Papadimitratos P, LA Fortelle A, Evenssen K, Brignolo R, Cosenza S (2009) Vehicular communication systems: enabling technologies, applications, and future outlook on intelligent transportation. IEEE Commun Mag 11:84-95

2. Chang SH, Lin CY, Hsu CC, Fung CP, Hwang JR (2009) The effect of a collision warning system on the driving performance of young 
drivers at intersections. Transport Res F: Traffic Psychol Behav 12(5):371-380

3. Chen H, Cao L, Logan DB (2011) Investigation into the effect of an intersection crash warning system on driving performance in a simulator. Traffic Inj Prev 12(5):529-537

4. Berndt H, Wender S, Dietmayer K (2007) Driver braking behavior during intersection approaches and implications for warning strategies for driver assistant systems. In: Intelligent Vehicles Symposium IEEE 2007:245-251

5. Karagiannis G, Altintas O, Ekici E, Heijenk G, Jarupan B, Lin K, Weil T (2011) Vehicular networking: a survey and tutorial on requirements, architectures, challenges, standards and solutions. IEEE Communications Surveys \& Tutorials 13:584-616

6. Lin Y-W, Chen Y-S, Lee S-L (2010) Routing protocols in vehicular ad hoc networks: a survey and future perspectives. J Inf Sci Eng 26(3):913-932

7. Young-Bae K, Vaidya NF (1998) Geocasting in mobile ad hoc networks: location-based multicast algorithms. Mobile Computing Systems and Applications, Proceedings. WMCSA '99. Second IEEE Workshop, pp 101-110

8. Michoud, R., Orozco, A., Llano, G. (2012) Mobile Ad-hoc routing protocols survey for the design of VANET applications. In: Intelligent Transportation Systems Symposium (CITSS), 2012. IEEE Colombian. pp 1-6

9. Li F, Wang Y (2007) Routing in vehicular ad hoc networks: a survey. Vehicular Technology Magazine, IEEE 2(2):12-22

10. Maihofer C (2004) A survey of geocast routing protocols. IEEE Communications Surveys Tutorials 6(2):32-42

11. Kihl M, Sichitiu M, Joshi HP (2008) Design and evaluation of two geocast protocols for vehicular ad-hoc networks. Journal of Internet Engineering 2(1)

12. IEEE Standard for Wireless access in vehicular environments WAVE - Networking services. IEEE Std 1609.3-2010 (Revision of IEEE Std 1609.3-2007). pp 1-144 (2010)

13. Jiang, Daniel, and Luca Delgrossi (2008) IEEE 802.11 p: Towards an international standard for Wireless Access in Vehicular Environments. Vehicular Technology Conference, 2008. VTC spring 2008. IEEE
14. Stancil DD, Bai F, and Cheng L (2010) Communication Systems for Car-2-X Networks. Vehicular Networking: automotive applications and beyond. John Wiley \& Sons, Ltd, pp 45-81

15. Krishnan H, Bai F, and Holland G (2010) Commercial and Public Use Applications. Vehicular Networking: Automotive Applications and Beyond. John Wiley \& Sons, Ltd, pp 1-28

16. S. Allal and S. Boudjit (2012) Geocast routing protocols for vanets: survey and guidelines. In: Innovative Mobile and Internet Services in Ubiquitous Computing (IMIS), 2012 Sixth International Conference on. pp 323-328

17. Allal S, Boudjit S (2013) Geocast routing protocols for VANETs: survey and geometry-driven scheme proposal. Journal of Internet Services and Information Security (JISIS) 3(1/2):20-36

18. Dressler F, Sommer C, Eckhoff D, Tonguz O (2011) Toward realistic simulation of intervehicle communication. IEEE Veh Technol Mag 6:43-51

19. Sommer C, German R, Dressler F (2011) Bidirectionally coupled network and road traffic simulation for improved IVC analysis. IEEE Trans Mob Comput 10:3-15

20. Behrisch, Michael, et al. (2011) SUMO-Simulation of Urban MObility. The Third International Conference on Advances in System Simulation (SIMUL 2011), Barcelona, Spain

21. Varga, András (2001) The OMNeT++ discrete event simulation system. Proceedings of the European simulation multiconference (ESM'2001). vol. 9 no. 185 sn

22. S. Krauss, P. Wagner, and C. Gawron (1997), Metastable states in a microscopic model of traffic flow. Phys Rev E 55(5) pp 5597

23. Ma C, Liu N (2013) Traffic-aware data delivery scheme for urban vehicular sensor networks. International Journal of Distributed Sensor Networks

24. X. Ma, X. Chen, and H. H. Refai (2008) On the broadcast packet reception rates in one-dimensional MANET. In: Global Telecommunications Conference, 2008. IEEE GLOBECOM 2008. IEEE, pp 1-5

25. F. Khan, Y. Chang, S. Park, and J. Copeland (2012) Towards guaranteed delivery of safety messages in VANET. In: Global Communications Conference (GLOBECOM), 2012 IEEE. pp 207-213 